\author{
Özen Özer ${ }^{1}$, Saleh Omran ${ }^{2,3}$ \\ ${ }^{1}$ Department of Mathematics, Faculty of Science and Arts, Kirklareli University, 39100, Turkey; \\ ${ }^{2}$ Department of Mathematics, Faculty of Science, Taif University, Saudi Arabia; \\ ${ }^{2}$ Department of Mathematics, Faculty of Science, South Valley University, Egypt \\ (E-mail: ozenozer39@gmail.com)
}

\title{
On the $C^{*}$-algebra valued $\mathrm{G}$-metric space related with fixed point theorems
}

\begin{abstract}
There are many fixed point results in the various kinds of metric spaces such as b-metric spaces, quaternion metric spaces, G-metric spaces, uniform spaces, non-commutative Banach spaces etc. In this work, we consider the one of modern methods as $C^{*}$-algebra G-metric space with fixed point theory to solve problems above mentioned. We prove the fixed point theorems for a mapping under the contractive conditions in $C^{*}$-algebra $G$-metric space. Besides, we establish the not only existence but also uniqueness theorem of fixed point in the such space. Also, we provide several examples to put support behind our main result.
\end{abstract}

Keywords: $G$-metric space, fixed point theory, $C^{*}$-algebra, banach contraction principle.

\section{Introduction}

Chandok et all. [1] introduced the concept of $C^{*}$-algebra valued partial metric and had illustrated examples significant fixed point results with provided examples. Dixmier [2] prepared his book includes in general $C^{*}$-Algebra with outstanding structures and theorems such as von Neumann, Stone-Weierstrass, Repr esentation and Duality theorems etc.

Kalaivani and Kalpana [3] considered the notion of $C^{*}$-algebra valued $b$ - metric spaces and obtained new contraction mapping gives an application to linear equation systems using such space. Kamran and his collabrates [4] generalized the new Banach contraction principle in $C^{*}$-algebra-valued $b$-metric and obtained some result for an integral equation as application in a $C^{*}$-algebra-valued $b$-metric space. Kang et all. [5] worked on the Complex valued $G$-metric spaces and also demonstrated and new contraction principle in such space. Zhenhua, Jiang and Sun [6] established the notion of $C *$-algebra-valued metric spaces and authenticated several fixed point with new idea in the set of a unital $C^{*}$-algebra's positive elements. Later, Omran and Salama [7] got some new resuts for common coupled fixed point theorems on $C^{*}$-algebra-valued metric spaces basic definitions and facts about structures of $C^{*}$-algebra and Fixed Point Theory.

G. Kalpana and C. Kalaivani [8] established coincidence fixed point theorems for two mappings in $C^{*}$-algebra-valued $S$-metric spaces which satisfy new contractive conditions. They demonstrated a common fixed point theorem for Banach contraction principle in this space. A. Zada et al. [9] considered graph theory and studied on common fixed points in the $C^{*}$-valued metric space endowed with the graph $G$ under $G$-contractive condition. S. Radenovic and his collegues [10] used a method to reducing coupled fixed point results to the respective ones for mappings with one variable in the framework of $b$-metric spaces. They also demonstrated that each $C^{*}$-algebra-valued $b$-metric space is a cone $b$-metric space over normal cone with normal constant equal to 1. The purpose of the paper Malhotraa \& Bansal [11] was to study on the the existence of the common coupled fixed point as well as uniqueness in complete $b$-metric spaces by considering diverse contractive conditions. Concept of generalized metric spaces (i.e. $G$-metric spaces) was introduced by Mustafa \& Sims [12] in and valuable results for fixed point theory in $G$-metric spaces were obtained by these authors. In their papers, Ozer \& Omran [13-15] have obtained useful and various types of fixed point theorems play a central role in the $C^{*}$-algebra valued metric spaces or $C^{*}$-algebra valued b-metric spaces. Shen, Jiang and Zhenhua [16] have proved new fixed-point theorems for self-mappings with contractive by introducing the notion of the complete $C^{*}$-algebra-valued G-metric space and also provided an application of the theory for a type of differential equations.

Similar work was done in [17] too. 
Definition 1.1. Let $\mathrm{X}$ be a non empty set and $G: X \times X \times X \rightarrow \mathbb{R}^{+}$, be a function satisfying the following properties:

$$
\begin{aligned}
& (G 1) G(e, f, g)=0 \quad \text { if } \quad e=f=g, \\
& \left(G_{2}\right) G(e, e, f)>0 \quad \text { for all } e, f \in X \text {, with } e \neq f, \\
& \left(G_{3}\right) G(e, e, f) \leq G(e, f, g) \quad \text { for all } e, f, g \in X \text { with } f \neq g \text {, }
\end{aligned}
$$

$(G 4) G(e, f, g)=G(e, g, f)=G(f, e, g)=G(f, g, e)=G(g, e, f)=G(g, f, e)$ (symmetry in all variables)

$$
\left(G_{5}\right) G(e, f, g) \leq G(e, k, k)+G(k, f, g) \quad \text { for all } k, e, f, g \in X . \text { (rectangle inequality) }
$$

Then the function $G$ is called as a Generalized Metric, more specifically a $G$-metric on $X$ and the pair $(X, G)$ is called a $G$-metric space.

Lemma 1.2. Let $\mathcal{A}$ be a $C^{*}$ - algebra and $a \in \mathcal{A}^{+}$. Then,

1) There is a unique element $b \in \mathcal{A}^{+}$such that $b^{2}=a$.

2) The set $\mathcal{A}^{+}=a^{*} a \mid a \in \mathcal{A}$ with a conjugate-linear involution $*: \mathcal{A} \rightarrow \mathcal{A}$

3) If $a, b \in \mathcal{A}$ and $0 \leq a \leq b$, then $\|a\| \leq\|\mathrm{b}\|$.

4) If $a \in \mathcal{A}^{+}$and $\|a\|<\frac{1}{2}$ then $(1-a)$ invertible and $\left\|\frac{a}{1-a}\right\|<1$.

On following references, the $C^{*}$ - algebra valued metric space is defined as follows:

Definition 1.3. Let $\mathrm{X}$ be a nonempty set and the mapping $\mathrm{d}: \mathrm{X} \times \mathrm{X} \rightarrow \mathcal{A}$ satisfies:

1) $0 \leq d(a, b)$ for all $a, b \in X$ and $0=d(a, b) \Leftrightarrow a=b$

2) $d(a, b)=d(b, a)$ for all $a, b \in X$

3) $d(a, b) \leq d(a, c)+d(c, b)$ for all $a, b, c \in X$

Then $\mathrm{d}$ is called a $C^{*}$-algebra valued metric on $\mathrm{X}$ and $(X, \mathcal{A}, d)$ is called $C^{*}$-algebra valued metric space.

Now, we introduce to $C^{*}$-algebra valued G- metric space as follows:

Definition 1.4. Let $\mathrm{X}$ be a nonempty set, and $G_{\mathcal{A}}: X \times X \times X \rightarrow \mathcal{A}$ be a mapping satisfying the following properties:

$$
\begin{gathered}
(G 1) G_{\mathcal{A}}(p, q, r)=0 \text { if } p=q=r, \\
\left(G_{2}\right) 0<G_{\mathcal{A}}(p, p, q), \quad \forall p, q \in X, \quad \text { with } \quad p \neq q,
\end{gathered}
$$

$\left(G_{3}\right) G_{\mathcal{A}}(p, p, q) \leq G_{\mathcal{A}}(p, q, r), \quad \forall p, q, r \in X \quad$ with $\quad p \neq r,\left(G_{4}\right) G_{\mathcal{A}}(p, q, r)=G_{\mathcal{A}}(p, r, q)=G_{\mathcal{A}}(q, p, r) \ldots$ (symmetry in all variables)

$$
\left(G_{5}\right) G_{\mathcal{A}}(p, q, r) \leq G_{\mathcal{A}}(p, k, k)+G_{\mathcal{A}}(k, q, r), \forall k, p, q, r \in X . \quad \text { (rectangle inequality) }
$$

Then the function $G_{\mathcal{A}}$ is called a $C^{*}$-algebra valued $\mathrm{G}$ - metric and the triple $\left(X, \mathcal{A}, G_{\mathcal{A}}\right)$ is called a $C^{*}$-algebra valued G- metric space.

Example 1.5. Let $X=\mathbb{R}$ and defined $G: X \times X \times X \rightarrow \mathcal{A}^{+}$by

$$
G(r, s, t)=\|r-s\| I+\|s-t\| I+\|t-r\| I
$$

for all $r, s, t \in X$ and $I$ is the unit element in $\mathcal{A}$. Then, $(X, \mathcal{A}, G)$ is a $C^{*}$-algebra valued $\mathrm{G}$ - metric space.

Definition 1.6. Let $(X, \mathcal{A}, G)$ be $\mathrm{C}^{*}$-algebra valued $\mathrm{G}$ - metric space and let $\left\{a_{n}\right\}$ be a sequence in $X$. We say that $\left\{a_{n}\right\}$ is convergent with respect to $\mathcal{A}$ and $a \in X$ if for any $e>0$, there exist $\exists n, m \in \mathbb{N}$ such that $G\left(a, a_{m}, a_{n}\right)<e$. We can also use different presentations for that as follows:

$a_{n} \rightarrow a$ or $\lim _{n \rightarrow \infty} G\left(a, a_{n}, a_{m}\right)=0$ or $\lim _{n \rightarrow \infty} a_{n}=a$

Definition 1.7. Let $(X, \mathcal{A}, G)$ be a $C^{*}$-algebra valued G- metric space and let $\left\{c_{n}\right\}$ be a sequence in $X$. The sequence $c_{n}$ is called as Cauchy sequence respect to $\mathcal{A}$ if, for $e>0$ there exists a positive integer $n^{*} \in N$ such that $G\left(c_{l}, c_{m}, c_{n}\right)<e, \forall n, l, m \geq n^{*}$ or $G\left(c_{l}, c_{m}, c_{n}\right) \rightarrow 0$ as $l, m, n \rightarrow \infty$ or $\left\|G\left(c, c_{m}, c_{n}\right)\right\| \rightarrow 0$ with $l, m, n \rightarrow \infty$.

We say $(X, \mathcal{A}, G)$ is complete $C^{*}$-algebra valued $\mathrm{G}$ - metric space if every sequence with respect to $\mathcal{A}$ is convergent. 


\section{Main Theorem and Results}

Proposition 2.1. Let $(X, \mathcal{A}, G)$ be a $C^{*}$-algebra valued G- metric space. Then for any $c \in X$, the followings are equivalent:

1. $c_{n}$ is a Cauchy sequence convergent to $c \in X$.

2. $\left\|G\left(c_{n}, c_{n}, c\right)\right\| \rightarrow 0$ as $n \rightarrow \infty$.

3. $\left\|G\left(c_{m}, c, c\right)\right\| \rightarrow 0$ as $m \rightarrow \infty$.

4. $\left\|G\left(c_{m}, c_{n}, c\right)\right\| \rightarrow 0$ as $m, n \rightarrow \infty$.

The proof can be done from the Definition 1.3.

Proposition 2.2. Let $(X, \mathcal{A}, G)$ be $C^{*}$-algebra valued G-metric space. Then the function $G(x, y, z)$ continues in all triple of values.

Proof. Suppose that let $\left\{x_{k}\right\},\left\{y_{m}\right\},\left\{z_{n}\right\}$ be convergent to $x, y, z \in X$ respectively. By $\left(G_{5}\right)$, we have

$$
\begin{aligned}
& G(x, y, z) \leq G\left(y, y_{m}, y_{m}\right)+G\left(y_{m}, x, z\right) \\
& G\left(x, z, y_{m}\right) \leq G\left(x, x_{k}, x_{k}\right)+G\left(x_{k}, y_{m}, z\right) \\
& G\left(x, z, y_{m}\right) \leq G\left(x, x_{k}, x_{k}\right)+G\left(x_{k}, y_{m}, z\right) \text { and } G\left(x, z_{k}, y_{m}\right) \leq G\left(z, z_{n}, z_{n}\right)+G\left(z_{n}, z_{m}, x_{k}\right) \\
& G(x, y, z)-G\left(x_{k}, y_{m}, z_{n}\right) \leq G\left(y, y_{m}, y_{m}\right)+G\left(x, x_{k}, x_{k}\right)+G\left(z, z_{n}, z_{n}\right) \\
& \Rightarrow\left\|G(x, y, z)-G\left(x_{k}, y_{m}, z_{n}\right)\right\| \leq\left\|G\left(y, y_{m}, y_{m}\right)\right\|+\left\|G\left(x, x_{k}, x_{k}\right)\right\|+\left\|G\left(z, z_{n}, z_{n}\right)\right\|,
\end{aligned}
$$

So,

since $\left\{x_{k}\right\},\left\{y_{m}\right\},\left\{z_{n}\right\}$ are convergent, this yields that

$\left\|G(x, y, z)-G\left(x_{k}, y_{m}, z_{n}\right)\right\| \rightarrow 0$ for $k, m, n \rightarrow \infty$.

Now, we prove the contraction principle $C^{*}$-algebra valued G- metric space as follows:

Theorem 2.3. Let $(X, \mathcal{A}, G)$ be a complete $C^{*}$-algebra valued $\mathrm{G}$ - metric space. Let $T: \quad X \rightarrow X$ be a contraction mappng on $\mathrm{X}$ such that

$$
G(T x, T y, T z) \leq a^{*} G(x, y, z) a,
$$

for all $x, y, z \in X$ where $a \in \mathcal{A}$ and $\|a\|<1$. So, contraction mapping $T$ has a unique fixed point.

Proof. Suppose that $T$ satisfies (1). Let $x_{0}$ be an arbitrary point in $X$ and define the sequence $x_{n}$ as follows:

$$
x_{1}=T x_{0}, x_{2}=T x_{1}, \ldots, x_{m}=T^{n} x_{0} .
$$

Then by (1), we get

$$
G\left(x_{n+1}, x_{n+1}, x_{n}\right)=G\left(T x_{n}, T x_{n}, T x_{n-1}\right) \leq a^{*} G\left(x_{n}, x_{n}, x_{n-1}\right) a .
$$

Again by (1), we get

$$
\begin{gathered}
G\left(x_{n+1}, x_{n+1}, x_{n}\right) \leq\left(a^{*}\right)^{2} G\left(x_{n-1}, x_{n-1}, x_{n-2}\right) a^{2} \\
\leq \ldots \\
\leq \ldots \\
\leq\left(a^{*}\right)^{n} G\left(x_{1}, x_{1}, x_{0}\right) a^{n} .
\end{gathered}
$$

Using Lemma 1.2, we have

$$
\begin{gathered}
\left\|G\left(x_{n+1}, x_{n+1}, x_{n}\right)\right\| \leq\left\|\left(a^{*}\right)^{n} G\left(x_{1}, x_{1}, x_{0}\right) a^{n}\right\| \\
\leq\left\|\left(a^{*}\right)^{n}\right\| \cdot\left\|G\left(x_{1}, x_{1}, x_{0}\right)\right\| \cdot\left\|a^{n}\right\|,
\end{gathered}
$$

since $\left\|\left(a^{*}\right)^{n}\right\|=\left\|a^{*} \cdot a^{*} \ldots . a^{*}\right\| \leq\left\|a^{*}\right\| \cdot\left\|a^{*}\right\| \ldots \ldots\left\|a^{*}\right\|$, we get

$$
\left\|a^{*}\right\|^{n} \cdot\|a\|^{n} \cdot\left\|G\left(x_{1}, x_{1}, x_{0}\right)\right\|=\|a\|^{2 n} \cdot\left\|G\left(x_{1}, x_{1}, x_{0}\right)\right\|
$$

due to $\left\|a^{*}\right\|=\|a\|$. 
Now, for $n>m$

$$
\begin{gathered}
G\left(x_{n}, x_{n}, x_{m}\right) \leq G\left(x_{n-1}, x_{n-1}, x_{n-2}\right)+G\left(x_{n-2}, x_{n-2}, x_{n-3}\right)+G\left(x_{n-3}, x_{n-3}, x_{n-4}\right) \\
\leq G\left(x_{m+1}, x_{m+1}, x_{m}\right)
\end{gathered}
$$

Therefore,

$$
\left\|G\left(x_{n}, x_{n}, x_{m}\right)\right\| \leq\left(\|a\|^{2 n}+\|a\|^{2 n-2}+\cdots+\|a\|^{2 m}\right)\left\|G\left(x_{1}, x_{1}, x_{0}\right)\right\| .
$$

For $n \rightarrow \infty$,

$$
\begin{aligned}
\left\|G\left(x_{n}, x_{n}, x_{m}\right)\right\| \leq & \|a\|^{2 m} \cdot\left(1+\|a\|+\|a\|^{2}+\ldots\right) \cdot\left\|G\left(x_{1}, x_{1}, x_{0}\right)\right\| . \\
& \leq \frac{\|a\|^{2 n}}{1-\|a\|} \cdot\left\|G\left(x_{1}, x_{1}, x_{0}\right)\right\| \rightarrow 0
\end{aligned}
$$

as $n, m \rightarrow \infty$. Thus, $x_{n}$ is a Cauchy sequence in $X$ with respect to $\mathcal{A}$ (by Proposition 2.1).

Next we prove that $x=T x$. Assume that $T x \neq x$, then by Proposition 2.1, we have

$$
G\left(T x_{n}, T x, T x\right) \leq G\left(x_{n+1}, T x, T x\right) \leq a^{*} G\left(x_{n}, x, x\right) a .
$$

Using Lemma 1.2, we obtain

$$
\begin{gathered}
0 \leq\left\|G\left(x_{n+1}, T x, T x\right)\right\| \leq\left\|a^{*}\right\| \cdot\|G(x, x, x)\| \cdot\|a\| \\
\leq\|a\|^{2} \cdot\left\|G\left(x_{n}, x, x\right)\right\| .
\end{gathered}
$$

Since $x_{n}$ is a Cauchy sequence then $\left\|G\left(x_{n}, x, x\right)\right\| \rightarrow 0$ as $n \rightarrow \infty$. Therefore, $\left\|G\left(x_{m+1}, T x, T x\right)\right\| \rightarrow 0, x_{n}$ convergences to $x$.

Thus $\left\|G\left(x_{n+1}, T x, T x\right)\right\| \rightarrow\|G(x, T x, T x)\|$, since $x_{n}$ is a Cauchy sequence and $\left.\| G x, T x, T x\right) \| \rightarrow 0$ by proposition 2.2 .

We prove that $x=T x$. To see that the fixed point is unique suppose that $x \neq y$ such that $T x=x, T y=y$. Then by (1) we have,

$$
0 \leq G(x, y, y)=G(T x, T y, T y) \leq a^{*} G(x, y, y) a .
$$

Therefore,

$$
0 \leq a^{*} G(x, y, y) a \leq\left\|a^{*}\right\| \cdot\|G(x, y, y)\| \cdot\|a\| .
$$

Put $\|a\|^{2}=k \in[0,1]$

$$
0 \leq\|G(x, y, y)\| \leq k \cdot\|G(x, y, y)\| .
$$

This is contradiction and we get

$$
\|G(x, y, y)\|=0 .
$$

So, $G(x, y, y)=0$ and this gives that $x=y$.

Example 2.4. Let $=\mathbb{R}, \mathcal{A}=\mathrm{M}_{3}(\mathbb{R})$ with $\|(p, q, r)\|=\max |r|,|q|,|p|$ (or $\mathcal{A}=\mathbb{R}^{3}$ with $\left.\|(p, q, r)\|=\left(|p|^{2}+|q|^{2}+|r|^{2}\right)^{\frac{1}{2}}\right)$ and $G(p, q, r)=\operatorname{dig}(|p-q|, a|q-r|,|p-r|)$ where $p, q, r \in X, \quad 0<a$, are constant. It is easy to check that $\left(X, \mathrm{M}_{3}(\mathbb{R}), G\right)$ is completed $C^{*}$-algebra valued G- metric space.

Proof. 1) It is clear that $G(p, q, r)>0$ holds.

$$
\begin{gathered}
\text { 2) if } p=q=r \Rightarrow G(p, q, r)=\operatorname{dig}\left(\begin{array}{lll}
0 & 0 & 0 \\
0 & 0 & 0 \\
0 & 0 & 0
\end{array}\right) . \\
G(p, p, q)=\operatorname{dig}\left(\begin{array}{ccc}
|p-p| & 0 & 0 \\
0 & a|p-q| & 0 \\
0 & 0 & |p-q|
\end{array}\right)=0_{\mathrm{M}_{3}(\mathbb{R})} \\
\text { with } p \neq q .
\end{gathered}
$$




$$
\begin{gathered}
G(p, p, q)=\operatorname{dig}\left(\begin{array}{ccc}
|p-p| & 0 & 0 \\
0 & a|p-q| & 0 \\
0 & 0 & |p-q|
\end{array}\right)=\operatorname{dig}\left(\begin{array}{ccc}
0 & 0 & 0 \\
0 & a|p-q| & 0 \\
0 & 0 & |p-q|
\end{array}\right) \\
=|p-q|\left(\begin{array}{ccc}
0 & 0 & 0 \\
0 & a & 0 \\
0 & 0
\end{array}\right)>0,(p \neq q, 0<a,) \\
\text { 3) } G(p, q, r)=\operatorname{dig}\left(\begin{array}{ccc}
|p-q| & 0 & 0 \\
0 & |q-r| & 0 \\
0 & 0 & |p-r|
\end{array}\right) \\
G(p, q, r)=\left(\begin{array}{cc}
|p-q| & 0 \\
0 & a|p-q|
\end{array}\right), a=1 \\
G(p, q, r)>0, p=q=r \Rightarrow G(p, q, r)=0
\end{gathered}
$$

and

$$
\begin{aligned}
& G(p, p, q)=\left(\begin{array}{cc}
|p-p| & 0 \\
0 & a|p-q|
\end{array}\right)>0 \text { if } u \neq v . \\
& G(p, p, q)=\left(\begin{array}{cc}
|p-p| & 0 \\
0 & 1|p-q|
\end{array}\right)<\left(\begin{array}{cc}
|p-q| & 0 \\
0 & 1|p-q|
\end{array}\right)=\left(\begin{array}{cc}
0 & 0 \\
0 & |p-q|
\end{array}\right)
\end{aligned}
$$

and since $G(p, q, r)=G(r, p, q)=\left(\begin{array}{cc}|r-p| & 0 \\ 0 & |p-q|\end{array}\right)$.

So,

$$
\left(\begin{array}{cc}
0 & 0 \\
0 & |p-q|
\end{array}\right)<\left(\begin{array}{cc}
|r-p| & 0 \\
0 & |p-q|
\end{array}\right)
$$

4) $G(p, q, r)=G(P(p, q, r))$ for all $P(p, q, r)$ points.

$$
\begin{gathered}
5) G(p, q, r)=\left(\begin{array}{cc}
|r-p| & 0 \\
0 & |p-q|
\end{array}\right)=\left(\begin{array}{cc}
|r-k+k-p| & 0 \\
0 & |q-k+k-r|
\end{array}\right) \\
\leq\left(\begin{array}{cc}
|r-k|+|k-p| & 0 \\
0 & |q-k|+|k-r|
\end{array}\right)=\left(\begin{array}{cc}
|k-p| & 0 \\
0 & 0
\end{array}\right)+\left(\begin{array}{cc}
|r-k| & 0 \\
0 & |q-k|
\end{array}\right)
\end{gathered}
$$

So,

$$
G(p, k, k)+G(r, k, q)=G(p, k, k)+G(k, q, r), \quad \forall k, p, q, r \in X .
$$

Example 2.5.

$X=L^{\infty}(E)$ and $H=L^{2}(E)$ where $E$ is Lebesgue space. by $\mathrm{B}(\mathcal{H})$ is the set of all bounded linear operators on $\mathcal{H}$. $\mathrm{B}(\mathcal{H})$ is $C^{*}$ algebra with operator norm

$$
\|\mathrm{T}\|=\sup a \in X,\|<T a, T a>\| .
$$

Define $G(f, g, h)=p_{|f-g|}+p_{|g-h|}, f, g, h \in X$ where $p_{h}: \mathcal{H} \rightarrow \mathcal{H}$ defined by $p_{h}(f)=h \diamond f$ composit of these operators where $h \in \mathcal{H}$ and $f \in H=L^{2}(E), p_{h}$ is the multiplacative operator.

Example 2.6. Let $X=[-1,1]$ and $\mathcal{A}=\mathbb{C}$ be complex.

$G(u, v, w)=\|u-v\| . k+\|v-w\| . k+\|w-u\| . k$ since $k>0_{\mathcal{A}}$, defined $T: X \rightarrow X$ such that $T x=x / 2$.

$$
G(T u, T v, T w)=\|u / 2-v / 2\| . I+\|v / 2-w / 2\| I+\|w / 2-w / 2\| . I
$$

$a=1 / 2,\|a\|=1 / 2<1$ and $\imath$ f we chose $k=I$ then $\imath$ t is clear that $\|I / 2\|=1 / 2 . I=\frac{1}{2}$.

Then, $T$ has a unique fixed point.

Remark. In the above example (Example 2.1) $X=L^{\infty}(E), H=L^{2}(E)$ and $\mathcal{A}=\mathrm{B}(\mathcal{H}) .(X, \mathcal{A}, G)$ defined above, let $G(f, g, h)=p_{|f-g|}+p_{|g-h|}$ and $T: L^{\infty}(E) \rightarrow(E)$ by

$$
\|G(T a, T b, T c)\|=\sup \mathrm{h} \in L^{2}(E),<p_{|\mathrm{Ta}-\mathrm{Tb}|} h, \mathrm{a}>+\sup \mathrm{h} \in L^{2}(E),<p_{|\mathrm{Tb}-\mathrm{Tc}|} h, \mathrm{a}>
$$


inner product in Hilbert space. Set,

$$
A=k . I,\|A\|<1 / 2 .
$$

(Following Zhenhu et all [10]),

We get

$$
\|G(T a, T b, T c)\| \leq\|k\|\left(\|a-b\|_{\infty}+\|b-c\|_{\infty}\right) \leq\|k\|\|G(a, b, c)\|
$$

and so, $T$ has a unique fixed point.

\section{Conclusion}

There is a good deal of applications for fixed point theory in many different fields of mathematics and engineering which include applied mathematics, dynamical systems, operation systems, Partial differential equations (PDE), ıntegral equations, computer sciences and also other scientific fields.

In this paper, we introduced the $\mathrm{C}^{*}$-Algebra valued $\mathrm{G}$ - metric space and established several important interesting/significant theorems for fixed point theory.

Results obtained in this paper useful for non commutative spaces to measure and operation system depended on PDE's to solve.

We hope that our results help the researchers to augment and also promote their studies on fixed point theory to carry out a general framework for the applications in life.

\section{References}

1 Chandok, S., Kumar, D., \& Park, C. C*-algebra valued partial metric space and fixed point theorems. ias.ac.in. Retrieved from https://www.ias.ac.in/public/Volumes/pmsc/forthcoming/PMSC-D-18-00162.$\mathrm{pdf}$

2 Dixmier, J. (1977). C*-Algebras". North-Holland Publ. Co., Amsterdam, New York, Oxford.

3 Kalaivani, C. \& Kalpana, G. (2018). A Fixed Point in $\mathrm{C}^{*}$-algebra-valued $\mathrm{S}_{b}$-metric spaces. Taga Journal, 14, 1442-1448.

4 Kamran, T., Postolach, M., Ghiura, A., Batul, S., \& Ali, R. (2016). The Banach contraction principle in $\mathrm{C}^{*}$-algebra valued b-metric spaces with application. Fixed Point Theory and Applications, 2016:10.

5 Kang, S.M., Singh, B., Gupta, V., \& Kumar, S. (2013). Contraction Principle in Complex Valued G-Metric Spaces. Int. Journal of Math. Analysis, 7(52), 2549-2556.

6 Zhenhua, M., Jiang, L., \& Sun, H. (2014). C*-algebra-valued metric spaces and related fixed point theorems. Fixed Point Theory and Applications, 2014:206.

7 Omran, S. \& Moustafa M. Salama (2018). Common Coupled Fixed Point in C*-algebras Valued Metric Spaces. International Journal of Applied Engineering Research ISSN 0973-4562, 13(8), 5899-5903.

8 Kalpana, G., \& Kalaivani, C. (2018). COMMON FIXED POINT THEOREMS IN C*-ALGEBRAVALUED S-METRIC SPACE. International Journal of Pure and Applied Mathematics, Vol. 119, No. 15, 367-375.

9 Zada, A., Saifullah, S. \& Ma, Z. (2016). COMMON FIXED POINT THEOREMS FOR G-CONTRACTION IN C*-ALGEBRA VALUED METRIC SPACES. International Journal of Analysis and Applications, Vol. 11, No. 1, 23-27.

10 Radenovic, S., Vetro, P., Nastasi, A., \& Quan, L.T. (2017). Coupled Fixed Point Theorems in C*-AlgebraValued b-Metric Spaces, SCIENTIFIC PUBLICATIONS OF THE STATE UNIVERSITY OF NOVI PAZAR, SER. A: APPL. MATH. INFORM. AND MECH., Vol. 9(1), 81-90.

11 Malhotra, N., \& Bansal, B. (2015). Some Coupled Common Fixed Point Theorems for Generalized Contraction in b-Metric Spaces. Journal of Nonlinear Analysis and Applications, 8, 8-16.

12 Mustafa, Z. \& Sims, B. (2006). A New Approach to a Generalize Metric Spaces. J. Nonlinear Convex Anal., 7, 289-297.

13 Özer, Ö., \& Omran, S. (2016). Common Fixed Point Theorems in C*- Algebra Valued b-Metric Spaces. AIP Conference Proceedings 1773, 050005. DOI: 10.1063/1.4964975. 
14 Özer, Ö., \& Omran, S. (2017). On The Generalized C*-Valued Metric Spaces Related With Banach Fixed Point Theory. International Journal of Advanced and Applied Sciences, 4(2), 35-37.

15 Özer, Ö., \& Omran, S. (2019). A Result On the Coupled Fixed Point Theorems in C*-algebra Valued b-Metric Spaces. Italian Journal of Pure and Applied Mathematics, No. 42, 722-730.

16 Shen, C., Jiang, L., \& Zhenhua, M. (2018). C*-Algebra-Valued G-Metric Spaces and Related Fixed-Point Theorems. Journal of Function Spaces, Article ID 3257189, 8.

17 Shehwar, D., \& Kamran, T. (2015). C*-Valued G-contractions and fixed points. Journal of Inequalities and Applications, 2015:304. DOI 10.1186/s13660-015-0827-9.

\author{
О. Озер, С. Омран
}

\title{
Қозғалмайтын нүкте жайлы теоремалармен байланысты $C^{*}$-алгебралық $G$-метрикалық кеңістік жайлы
}

\begin{abstract}
b-Метрикалық кеңістік, кватерниондық метрикалық кеңістік, $G$ - метрикалық, біркелкі, коммутативті емес банах және тағы басқа әртүрлі метрикалық кеңістіктерде нақтыланған нүктелі көптеген нәтижелер бар. Мақалада жоғарыда көрсетілген есептерді шешу үшін қозғалмайтын нүкте теориясымен $G$-метрикалық кеңістіктің $C^{*}$-алгебрасы сияқты қазіргі заманғы әдістердің бірі қарастырылды. $G$-кеңістігінің $C^{*}$-алгебрасында сығылмалы жағдайларда бейнелеу үшін қозғалмайтын нүкте жайлы теоремалар дәлелденді. Сонымен қатар тек қана бар болу теоремасын ғана емес, сондай-ақ осы кеңістіктегі қозғалмайтын нүктенің жалғыздығы жайлы теоремасын да нақтылады. Сонымен қатар негізгі нәтижені қолдаудың бірнеше мысалы келтірілді.
\end{abstract}

Kiлm сөздер: $G$-метрикалық кеңістік, қозғалмайтын нүктелер теориясы, $C^{*}$-алгебра, Банахтың сығылмалы бейнелеулер қағидасы.

\section{О. Озер, С. Омран \\ О $C^{*}$-алгебраическом $G$-метрическом пространстве, связанном с теоремами о неподвижных точках}

Существует множество результатов с фиксированной точкой в различных метрических пространствах, таких как b-метрические, кватернионные метрические, $G$-метрические, равномерные, некоммутативные банаховы пространства и т.д. В статье рассмотрен один из современных методов $C^{*}$-алгебра G-метрического пространства с теорией неподвижной точки для решения указанных выше задач. Доказаны теоремы о неподвижной точке для отображения при сжимающих условиях в $C^{*}$-алгебре $G$-метрического пространства. Кроме того, установлена не только теорема существования, но и теорема единственности неподвижной точки в таком пространстве. Также приведены примеры поддержки нашего основного результата.

Ключевые слова: $G$-метрическое пространство, теория неподвижных точек, $C^{*}$-алгебра, принцип сжимающих отображений Банаха. 